\title{
A Lesson Learned from Outer Billiard Derivatives
}

\author{
Samuel Otten" and Filiz Doğru \\ Department of Mathematics \\ Grand Valley State University \\ 1 Campus Drive \\ Allendale, Michigan 49401-6495, USA
}

Received: September 15, $2009 \quad$ Accepted: March 16, 2010

\begin{abstract}
As students move onward and upward through collegiate mathematics they are often impressed by the power of advanced techniques (aren't they?), techniques allowing problems that were previously difficult or near-impossible to be solved with relative ease. For example, once students have learned the residue theorem in complex analysis they are able to elegantly evaluate integrals that were unwieldy in calculus. Among practicing mathematicians there is also a tendency to look to new or powerful results when trying to unlock a problem within one's own research. This article presents a new theorem concerning derivatives within the outer billiard dynamical system, and in so doing serves as a reminder that higher-powered mathematics are not always needed and do not necessarily produce more satisfying proofs. Sometimes it is beneficial to keep elementary approaches in mind.
\end{abstract}

\section{INTRODUCTION TO OUTER BILLIARDS}

Outer billiards is a dynamical system that is emerging in popularity (see, for example, [3], [10] and [14]). It can be thought of both as a counterpart to the wellknown (inner) billiards system and as a crude model for planetary motion (as in [7] and [8]). An outer billiard system begins with a table and a ball, that is to say, a closed convex domain $P \subset \mathbb{R}^{2}$ and a point $x$ in the exterior of $P$. Provided that $x$ does not lie on the extension of a side of $P$, there are two points on $P$ that form support lines ${ }^{1}$ through $x$. The outer (or dual) billiard transformation-denoted $F$-has either a clockwise or a counterclockwise orientation and is defined as the map that sends $x$ to its reflection through the support point in the

\footnotetext{
1 Support lines and support points are generalizations of the concepts of tangent lines and tangent points, respectively. We use the more general notions because our tables are not always smooth.

\# Currently a graduate student at Michigan State University (ottensam@msu.edu)
}

given direction (see Figure 1). If $x$ does lie on an extension of a side of $P$, such that the support point is not unique, then $F$ is undefined, in much the same way that the inner billiard transformation is undefined when the ball hits a corner of the table. The set of all iterations of a particular point is called an orbit of $F$, and if $F^{k}(x)=x$ for some $k \in \mathbb{N}$ then we say that the point and orbit are $k$-periodic.

This setup is all that some mathematicians need to begin work within outer billiards. Indeed, many interesting articles exist concerning the periodic (or non-periodic) behavior of the map with respect to certain tables in the Euclidean plane (e.g., [5], [12], and [13], see Figure 2). Another active line of research has centered on the Moser-Neumann problem of whether or not outer billiard systems exist in which orbits are unbounded (e.g., [1], [2], [11]). For this article, however, we take a different approach by moving the outer billiard game to the hyperbolic plane where we do a bit of calculus. 

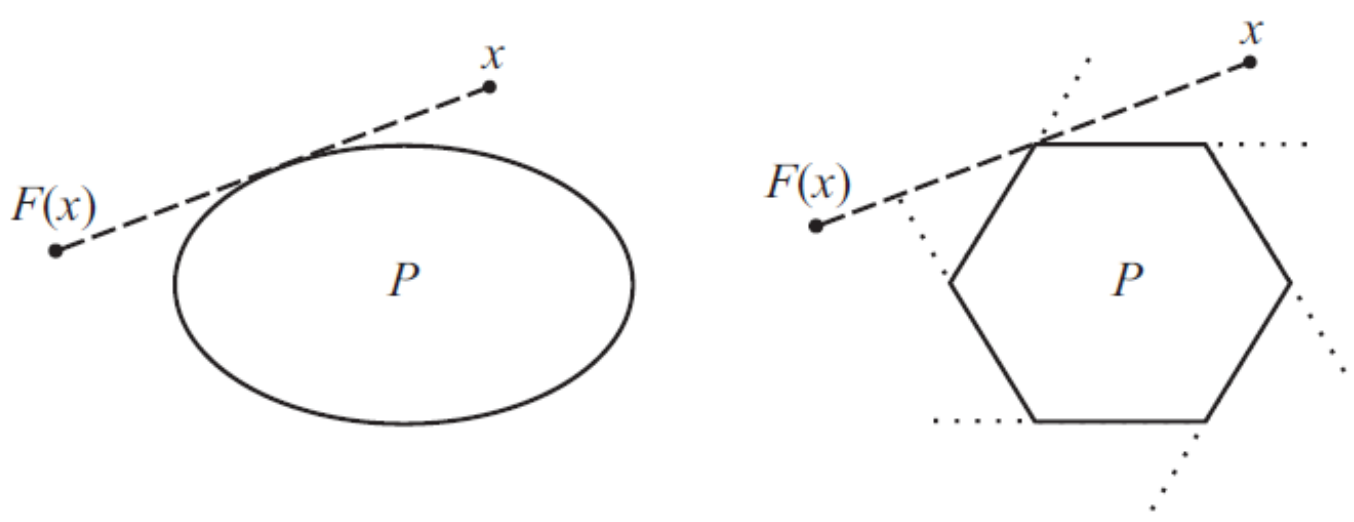

Figure 1. Outer billiard mappings with counterclockwise orientation.

\section{DERIVATIVES AT THE BOUNDARY OF THE HYPERBOLIC PLANE}

We employ the Beltami-Klein disk model of the hyperbolic plane, rather than the Poincaré disk model, because geodesics in the Beltami-Klein model are straight lines. This fact allows our (Euclidean) intuition to be used when dealing with collinear points and closed figures. These benefits outweigh the fact that the model is non-conformalthat is, unlike the Poincaré model, angle measurements in the Beltrami-Klein model are not the same as angle measurements in Euclidean space [4].

With this model in hand, we can define the outer billiard transformation $F$ in the hyperbolic plane by taking the Euclidean definition from above and replacing Euclidean distances with hyperbolic distances. Moreover, we can extend the map $F$ to be defined at infinity (i.e., on the boundary of the disk). The restriction of the extended outer billiard transformation to the boundary of the disk is denoted $f$, which gives a circle map at the boundary, $f: S^{1} \rightarrow S^{1}$. Let us write the derivative of $f$ at a periodic point $p$ on the boundary of the disk as $D_{f}(p)$. Since the tangent space to $S^{1}$ at $p$ identifies with $\mathbb{R}$, we know that $D_{f}(p)$ is a linear map from $\mathbb{R}$ to itself and so a real number. This number can be computed in the following way.
Let $x$ be a point in the tangent space of $p$ infinitesimally close to $p$, with $o$ a support point. Denote the image of $x$ as $f(x)$ and the image of $p$ as $f(p)$. Then, as depicted in Figure 3, we have

$$
D_{f}(p)=\lim _{x \rightarrow p} \frac{|f(x) f(p)|}{|x p|}
$$

The two infinitesimal triangles $\Delta p o x$ and $\Delta f(p) o f(x)$ are similar, thus

$$
\frac{|f(x) f(p)|}{|x p|}=\frac{b}{a}
$$

By continuing around the circle, and using the chain rule as needed, it follows that the derivative of $f$ at a $n$-periodic point $p$ on the boundary of the disk can be computed by

$$
D_{f}(p)=\prod_{i=1}^{n} \frac{b_{i}}{a_{i}},
$$

where $a_{i}$ is the Euclidean distance from $f^{i-1}(p)$ to the support point between itself and $f^{i}(p)$, and $b_{i}$ is the Euclidean distance from $f^{i}(p)$ to that same support point (see Figure 4). Readers are encouraged to examine the detailed yet accessible proof in [2].

There are a few things to note about this formula. First, the distances in the formula are indeed Euclidean even though 


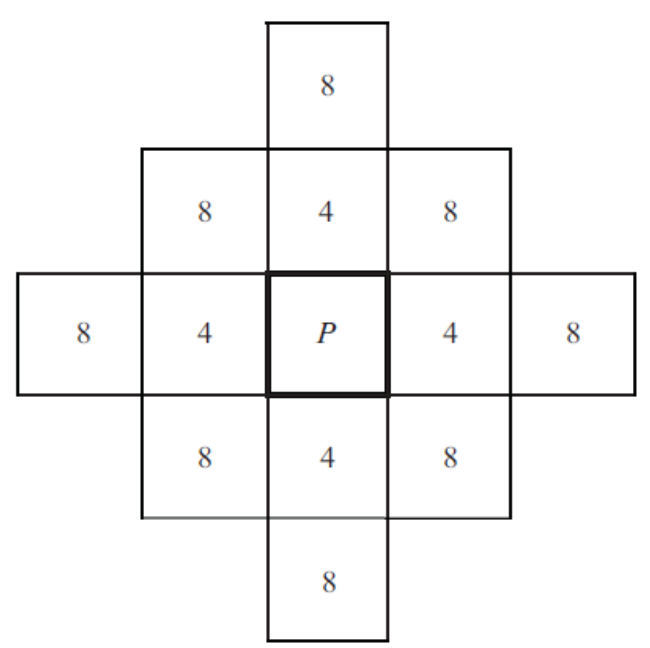

Figure 2. 4-periodic and 8-periodic regions about a square.

we are in the hyperbolic plane (hyperbolic distances in this situation are infinite). Second, there may be vertices of a polygonal table that are not support points of a periodic orbit at infinity and so contribute nothing to the derivative. This observation is related to the notion of a symbolic orbit, which is the tuple of vertices (with possible repetitions) comprising the support points of the given orbit. Symbolic vertices in the tuple-a sort of "support table" (see Figure 5). Third, the derivative as presented above is defined for a point, but one can verify that it is also well-defined with respect to an orbit at infinity. This works because the derivatives at all points from the same orbit are equal (as is clear from Figure 4), and the derivative for an orbit can be defined as the common value of $D_{f}(p)$. A natural question arises: What is the relationship between derivatives from different orbits at infinity around the same table?

Figure 6 depicts two 3-periodic orbits with the same symbolic orbit. (Per the remarks above, it does not make sense to compare different symbolic orbits because they are essentially orbits about different tables.) Empirical investigations ${ }^{2}$ of these

\footnotetext{
${ }^{2}$ The problem of finding the periodic orbits of $f$ about a polygonal table is still open. However, we know that in hyperbolic geometry, since the map is a composition of reflections, it is either parabolic, elliptic, or hyperbolic. Therefore, the map has periodic orbits at the boundary in addition to possible investigations, we often started with the orbits instead periodic orbits in the plane
}

and other orbits would lead to the conjecture that, for $x$ and $y$ from distinct $n$-periodic orbits at infinity, we have

$$
\begin{gathered}
D_{f}(x)=\frac{b_{1} b_{2} \ldots b_{n}}{a_{1} a_{2} \ldots a_{n}}=\frac{c_{1} c_{2} \ldots c_{n}}{d_{1} d_{2} \ldots d_{n}} \\
=\frac{1}{D_{f}(y)}
\end{gathered}
$$

For periodic points with the same support table, this reciprocal relationship does in fact hold generally-but how should we go about proving it?

\section{PROOFS OF RECIPROCITY}

Regarding the conjecture above, those readers who are familiar with particular pieces of "high-powered" mathematics-namely, isometry classifications in the hyperbolic plane and Möbius transformations-may already sense an avenue toward proof. We shall head in this direction first.

Let $P$ be an outer billiard table such that $f$ has two $n$-periodic points $x$ and $y$ from distinct orbits. (It is shown in [6] that

(depending on the size of the table). To find the orbits at the boundary for our empirical of the table or we ran the map in reverse, which revealed the periodic orbits. For more on the attractive and repulsive properties of these orbits, see [1]. 


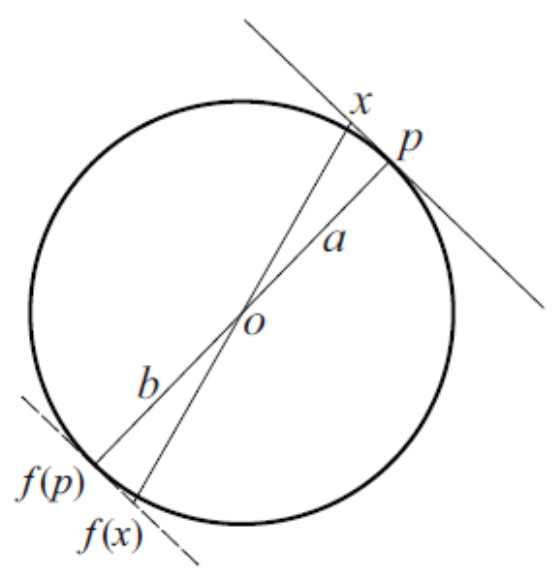

Figure 3. The derivative of $f$ at the point $p$.

periodic points of $f$ necessarily have the same period.) Suppose that the points from each of these two orbits form simple polygons (which circumscribe $P$ ). Suppose further that these two orbits have the same symbolic orbit $S$, which by the first supposition is necessarily an $n$-tuple with no repetitions. Then we can restrict the reflections of the points in the complement of $P$ to be just through these $n$ distinct vertices of $S$, and this will not alter the periodic orbits. By considering each of these $n$ reflections a separate outer billiard map, we can compose them to form a piecewise-continuous hyperbolic isometry of the hyperbolic plane and a continuous hyperbolic isometry of the boundary (because, by [2], there are two fixed points at infinity, namely, the orbits). Now, a hyperbolic isometry of this sort in the hyperbolic plane can be described by a Möbius transformation [4], and the two fixed points of the Möbius transformation are precisely the points $x$ and $y$. Since the derivatives at the fixed points of a Möbius transformation are reciprocals and will correspond with the derivatives of $f$, it follows that $D_{f}(x)=1 / D_{f}(y)$.

This argument is mathematically valid and contains an elegant connection between outer billiards and Möbius transformations. But what does it do for undergraduates who have yet to be exposed to this mathematical machinery? Let us turn to an elementary proof route that works just as well (if not better).

Theorem. Let $P$ be an outer billiard table in the hyperbolic plane such that the extended outer billiard map $f$ about $P$ has two periodic orbits at infinity with the same symbolic orbit. The points $x$ and $y$ are from distinct periodic orbit if and only if

$$
D_{f}(x)=\frac{1}{D_{f}(y)} \text {. }
$$

We prove this full theorem below using elementary geometry in the manner of [9]. Let us begin with the forward direction and assume that the points are $n$-periodic, writing $x_{i}$ for $f^{i}(x)$ and $y_{i}$ for $f^{i}(y), 0 \leq i \leq$ $n$. Note that by periodicity $x_{0}=x_{n}$ and $y_{0}=y_{n}$. Since derivatives of $f$ are pointindependent within an orbit, we are free to choose $x_{0}$ and label $y_{0}$ so that $x_{1}$ and label $y_{1}$ have reflected through the same support point and $x_{1}$ lies on the arc between $y_{0}$ and $y_{1}$ (see Figure 6, for example). With this labeling, the chord from $x_{i-1}$ to $x_{i}$ and the chord from $y_{i-1}$ to $y_{i}$ intersect at the support point $s_{i}$. By the two-chord theorem, the product of segments formed on one intersecting chord is equal to that on the

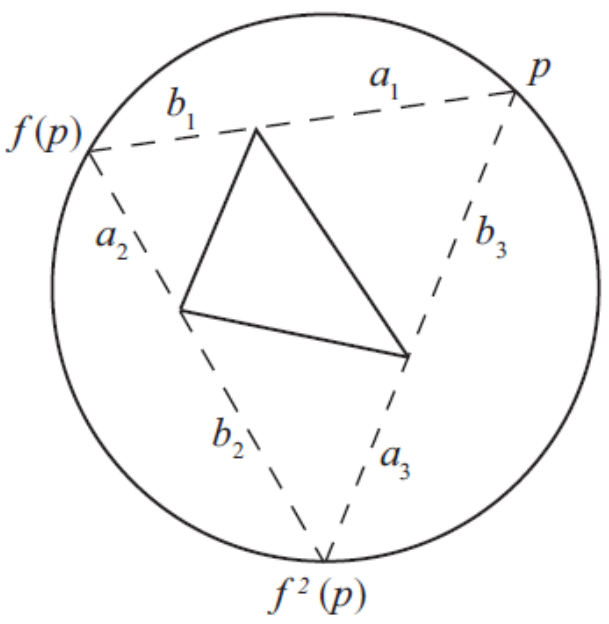

Figure 4. The derivative of $f$ at $p$ is the quotient of $b_{1} b_{2} b_{3}$ and $a_{1} a_{2} a_{3}$. 

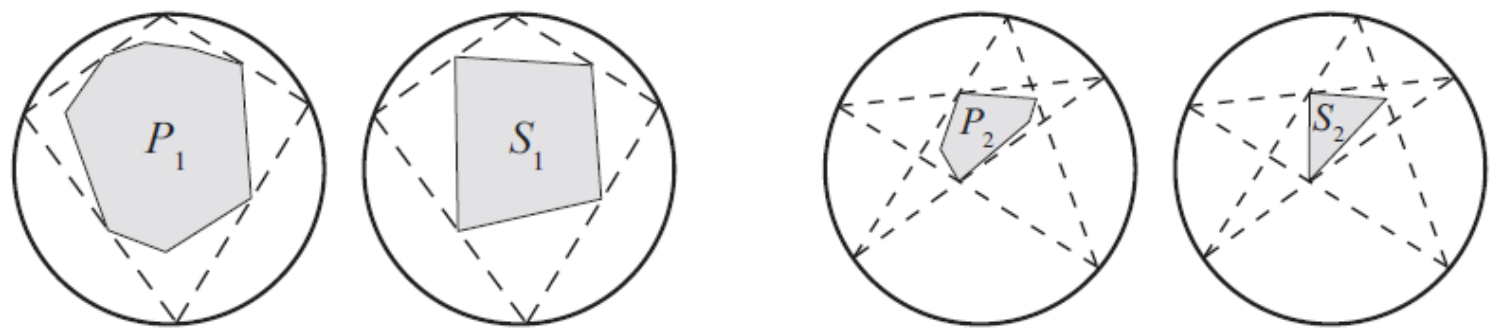

Figure 5. Original tables and informal symbolic orbits.

other chord. Using this at all such intersections, the product of the results is

$$
\prod_{i=1}^{n} a_{i} b_{i}=\prod_{i=1}^{n} c_{i} d_{i}
$$

where $a_{i}$ and $b_{i}$ correspond to the distances used in the derivative formula for $D_{f}(x)$ and $c_{i}$ and $d_{i}$ correspond to the distances used in the derivative formula for $D_{f}(y)$.

Since $x$ and $y$ are from distinct orbits, we know that $x_{i}, s_{i}$, and $y_{i-1}$ are never collinear. We therefore construct $n$ triangles using $x_{i}, s_{i}$, and $y_{i-1}$ as the vertices, and $b_{i}$ and $c_{i}$ are necessarily sides of these triangles (see Figure 7, for example). Looking within the triangles, $\angle s_{i} y_{i-1} x_{i}$ is equivalent to the inscribed angle with endpoints $x_{i}$ and $y_{i}$, call it $\theta_{i}$. Similarly, $\angle s_{i} x_{i} y_{i-1}$ will be called $\theta_{i-1}$. The Law of Sines gives $b_{i} \sin \left(\theta_{i}\right)=c_{i} \sin \left(\theta_{i}\right)$ for $0 \leq i \leq n$. We can multiply all of these together to arrive at

$$
\prod_{i=1}^{n} b_{i} \sin \left(\theta_{i-1}\right)=\prod_{i=1}^{n} c_{i} \sin \left(\theta_{i}\right) .
$$

We have $\sin \left(\theta_{i}\right)$ appearing on both sides of the equation for $0 \leq i \leq n$. As noted above, $x_{0}=x_{n}$ so $\theta_{0}=\theta_{n}$, and $\sin \left(\theta_{0}\right)=$ $\sin \left(\theta_{n}\right)$. Thus we may cancel all the same terms, leaving

$$
\prod_{i=1}^{n} b_{i}=\prod_{i=1}^{n} c_{i}
$$

Considering equation 2 together with equation 1 yields

$$
D_{f}(x)=\prod_{i=1}^{q} \frac{b_{i}}{a_{i}}=\prod_{i-1}^{q} \frac{c_{i}}{d_{1}}=\frac{1}{D_{f}(y)},
$$

and the forward direction is proven.

For the contrapositive of the backward direction, suppose that $x$ and $y$ are from the same orbit. Then $D_{f}(x)=$ $D_{f}(y)$. Since, by hypothesis, there are two periodic orbits on the circle, $f$ is not a parabolic isometry of the Beltrami-Klein disk.

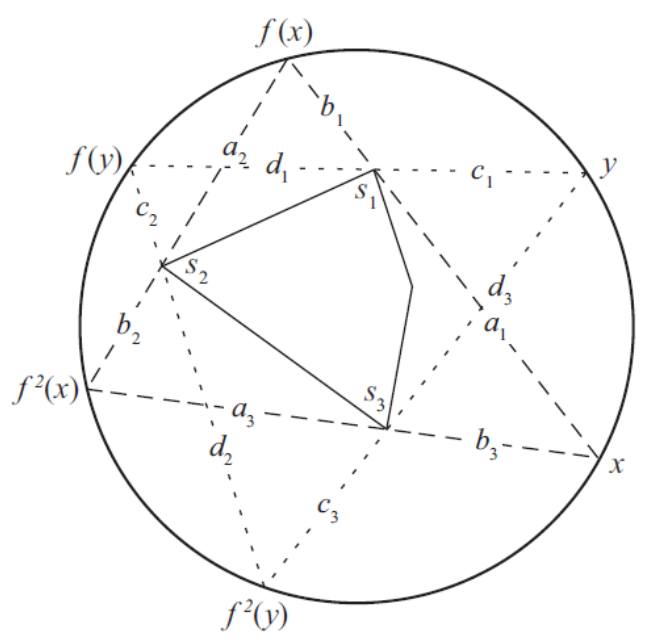

Figure 6. Two 3-periodic orbits about the same "support table." 


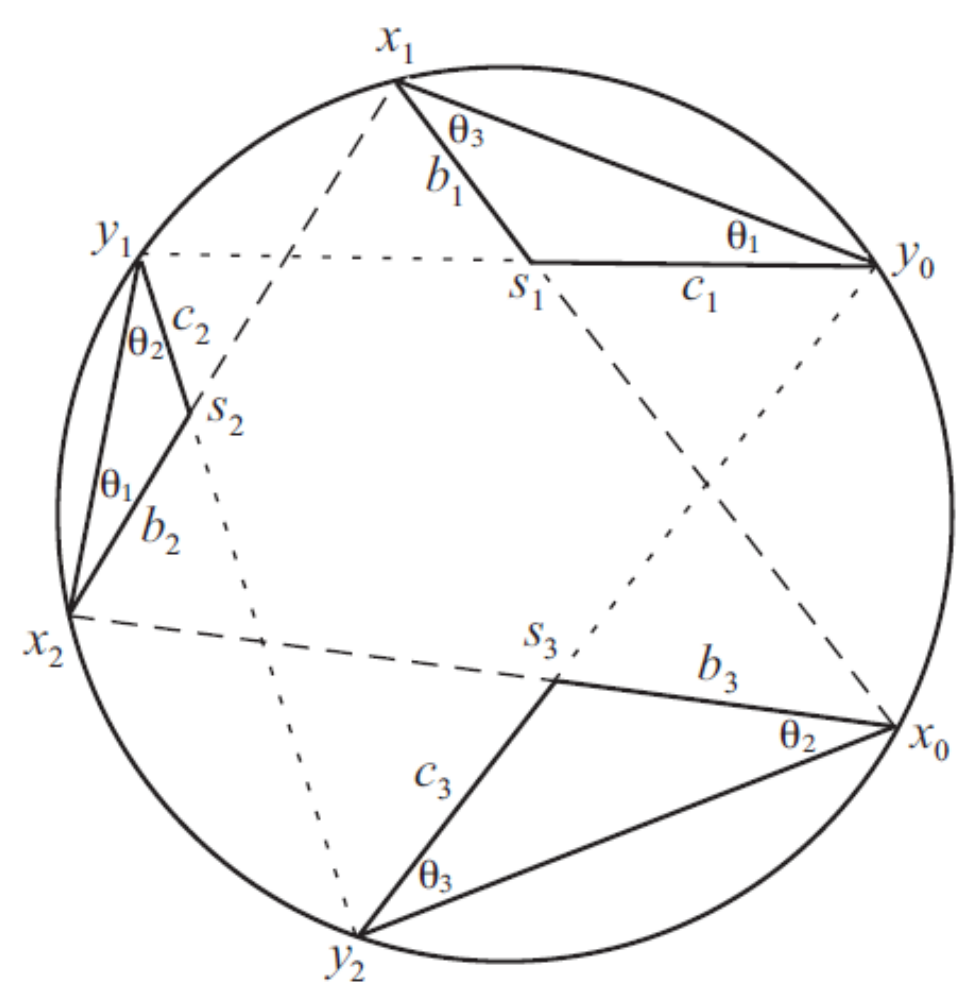

Figure 7. Triangles with corresponding angle measures.

Thus, by properties of isometries of the hyperbolic plane, the derivatives of $f$ at its periodic points are not 1 . So $D_{f}(x) \neq$ $1 / D_{f}(y) .^{3}$

Many would say that this proof using elementary geometry gives a more intuitive sense of why the theorem is true. Even if some would debate this point, it is not debatable that this geometric proof is more accessible to young mathematicians than the Möbius transformation proof. Moreover, the Möbius proof depends on the assumption that the two orbits form simple polygons, whereas it can be seen clearly in the geometric proof that the only requirement is equal symbolic orbits (i.e.,the argument is valid so long as the orbits

\footnotetext{
${ }^{3}$ Note that this theorem implies that there can only be two distinct periodic orbits about a given support table because a point $z$ from a third orbit would force $x$ and $y$ to be from the same orbit. This also follows from the (high-powered) fact that $f$ is a hyperbolic isometry and thus has two fixed points.
}

contain the same support points in the same order [9].)

\section{CONCLUSION}

We hope that this article has served three purposes. First, to offer a gentle introduction to the outer billiard dynamical system and a modest list of references for those who may be interested in investigating it further. Second, to uncover a reciprocal relationship between derivatives at periodic points of the extended outer billiard map. Third-and perhaps most importantly - to remind us that elementary methods can often be "over achieving" and that it is not always necessary to bring high-powered machinery to bear on mathematical problems.

\section{ACKNOWLEDGMENTS}

This work was supported in part by a professional development grant from the 
NSF Advance Paid grant at Grand Valley State University. We also thank the Student Summer Scholars program at Grand Valley State for its generous support.

\section{REFERENCES}

1. S. Otten and F. Doğru, "Sizing Up Outer Billiard Tables" Am. J. Undergrad. Res., to appear in September 2010.

2. F. Doğru and S. Tabachnikov, "On Polygonal Dual Billiard in the Hyperbolic Plane" Reg. Chaotic Dynamics 8 (2002) 67-82.

3. F. Doğru and S. Tabachnikov, "Dual Billiards" Math. Intelligencer. 27 (2005) 18-25.

4. M.J. Greenberg, Euclidean and NonEuclidean Geometry: Development and History (W.H., Freeman \& Co, New York, NY, 1993).

5. E. Gutkin and N. Simanyi,, "Dual Polygonal Billiards and Necklace Dynamics" Comm. Math. Physics 143 (1991) 431-450.
6. B. Hasselblatt and A. Katok, A First Course in Dynamics (Cambridge University Press, Cambridge [UK] and New York [USA], 1993).

7. J. Moser, "Is the Solar Sysytem Stable?" Math. Intelligencer 1 (1978) 65-71.

8. B. Neumann, "Sharing Ham and Eggs" lota, (Manchester [UK] University, 1959).

9. S. Otten, "Do cyclic polygons make the cut?" Math. Mag. 80 (2007) 138-141.

10. R. Schwartz, "Unbounded Orbits for Outer Billiards" J. Modern Dynamics 1 (2007) 371-424.

11. R. Schwartz, "Outer Billiards on Kites" arXiv monograph (2008).

12. S. Tabachnikov, "Billiards" (Soc. Math. De France, Paris, 1995).

13. S. Tabchnikov, "Asymptotic Dynamics of the Dual Billiard Transformation" J. Statistical Physics 83 (1996) 27-38.

14. S. Tabachnikov. "A Proof of Cutler's Theorem on the Existence of Periodic Orbits in Polygonal Outer Billiards" Geom. Dedic. 129 (2007) 83-87.

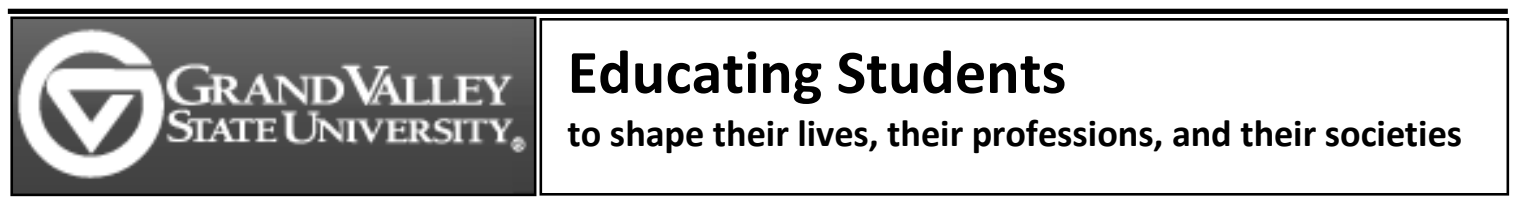

The Department of Mathematics at Grand Valley State University is devoted first and foremost to excellence in teaching, reflecting the goals of Grand Valley State University.

Our mission is to:

- teach mathematics so as to develop in our students

- an appreciation of mathematics as a driving force in society, culture and history,

- an understanding of mathematics and its applications, and

- the ability to reason and communicate mathematically

as well as to

- assimilate new mathematical ideas;

- engage in active scholarship, in mathematics and mathematics education, which supports our teaching and furthers mathematical understanding;

- build productive connections with academic and non-academic communities.

\section{http://www.gvsu.edu/math/index.html}




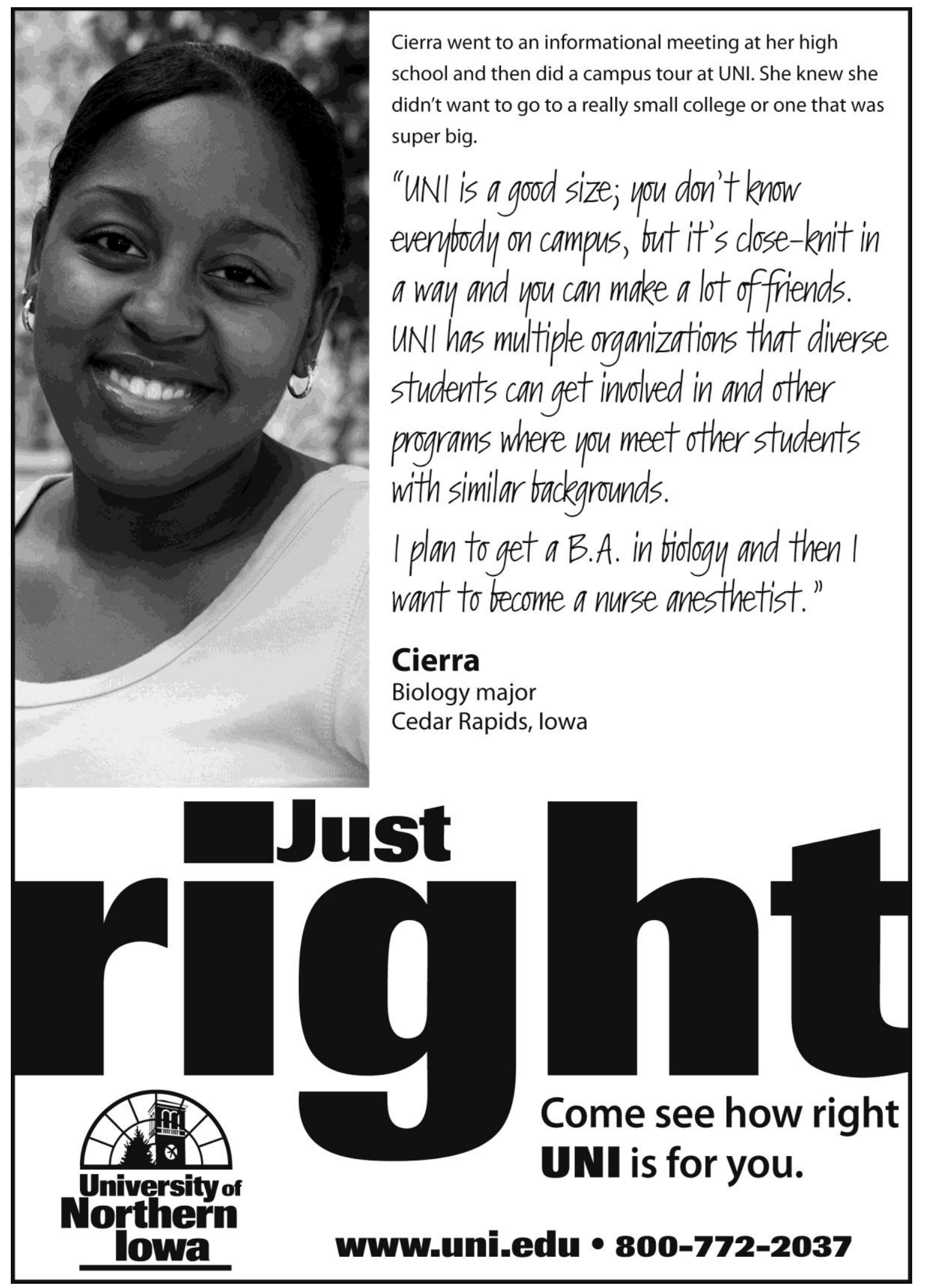

